\section{CHANGE OF TEAR OSMOLARITY AFTER REFRACTIVE SURGERY}

\section{SUMMARY}

\section{CHANGE OF TEAR OSMOLARITY AFTER REFRACTIVE SURGERY}

Purpose: To compare the influence of the LASIK and ReLEx SMILE refractive method on tear osmolarity

Methods: Prospective non-randomized comparative study. We evaluated the results in two groups of patients who underwent binocular refractive surgery to remove myopia and possibly astigmatism in the eye clinic of Horní Počernice. In each group were 15 patients (30 eyes), patients of one group undergoing FS-LASIK refractive surgery, in the second group a ReLEx SMILE procedure. Bilateral measurement of the tear film osmolarity using the TearLab instrument on the day of surgery, the first day after surgery, one month and 3 months after surgery was performed on each patient.

Results: The mean preoperative value of the osmolarity of the tear film was practically the same in both groups, in the ReLEx SMILE group $294.9 \pm 13.4 \mathrm{mOsm} / \mathrm{l}$ and in the FS-LASIK group $296.4 \pm 13.1$. One and three months after the surgery in the ReLEx SMILE group, these values increased to 301.4 and $296.4 \pm 13.3 \mathrm{mOsm} / \mathrm{l}$ respectively, the elevation of values one month after surgery was statistically significant. In the FS-LASIK group, one month after surgery, the osmolarity was $320.1 \pm 14.7$, three months postoperatively $306.5 \pm 13.1 \mathrm{mOsm}$ / I. Both of these values compared to the preoperative value were statistically significantly higher. The increase of the mean osmolarity was statistically significantly higher in the FS-LASIK group compared to the ReLEx SMILE. Three months after surgery, the difference between groups was not statistically significant.

Conclusion: In our group of patients, after the FS-LASIK refraction procedure, we noted a higher mean osmolarity of tear film in comparison with patients after the ReLEx SMILE in all evaluated timepoints ( 1 day, 1 month and 3 months after surgery). We consider ReLEX SMILE as a method with less impact on the quality of tear film, with a faster return to original osmolarity and potentially higher patient comfort.

Key words: Tear osmolarity, Relex SMILE, FS-LASIK

Čes. a slov. Oftal., 74, 2018, No.1 , p. 18-22
Kacerovská J. ${ }^{1}$, Kacerovský M. ${ }^{1}$,

Hlaváčková M. ${ }^{1}$, Studený P. ${ }^{2}$

${ }^{1}$ Eye Centre Horní Počernice, Prague,

Obchodní 2694/2, 19300 Prague 9, Horní

Počernice

Chief physician: MUDr. Richard Havránek

${ }^{2}$ Department of Ophthalmology,

Královské Vinohrady University Hospital

and 3rd Faculty of Medicine, Charles

University Prague, Šrobárova 50, Prague

10 - Vinohrady, 10034

Chief doc. MUDr. Pavel Studený, Ph.D.

MHA

The authors of the study declare that no conflict of interest exists in the compilation, theme and subsequent publication of this professional communication, and that it is not supported by any pharmaceuticals company.

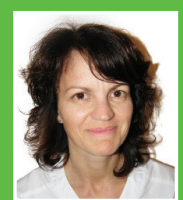

MUDr. Jana Kacerovská

Oční centrum Horní Počernice Praha

Obchodní 2694/2

19300 Praha 9, Horní Počernice

kacerovska@ocniklinikahp.cz

\section{INTRODUCTION}

Disorders of the lachrymal film and problems with dry eye are one of the frequent postoperative complications in patients who have undergone a LASIK (laser in situ keratomileusis) type refractive procedure $[19,24]$. It is stated that transitional symptoms in connection with dry eye are described by as many as $95 \%$ of patients who have undergone this procedure [24]. Although the precise mechanism of the complaints is not known, it is assumed that the fundamental cause is impairment of innervation of the cornea $[2,20]$. This fact is also linked to the reduced frequency of blinking and subsequent incidence of epitheliopathy [15]. Other possible factors that have been described include a reduction of the number of goblet cells caused by suction [18], chronic inflammation of the ocular surface [4], changes of corneal curvature and thus ensuing change of distribution of the lachrymal film [8].

The ReLEx SMILE method (refractive lenticule extraction, small incision lenticule extraction) is a relatively new type of refractive procedure, indicated primarily in patients with medium and higher myopia and if applicable also astigmatism $[1,16]$. In this operation femtosecond laser is used to create an intrastromal lenticule, which is released and removed from the cornea by a service approx. $3 \mathrm{~mm}$ large corneal incision. As a result of this, a change takes place of the curvature of the anterior surface of the cornea, as well as of the refractive state of the eye, without the use of an excimer laser. One of the stated advantages of this method in comparison with the FS -LASIK (femtosecond laser-assisted in situ keratomileusis) method is less disturbance of the innervation of the cornea, and thus also a reduction of the negative influence on the occurrence of complaints with dry eye. Although a breach of the nerve fibres takes place upon lamellar separation of the cornea in both methods, in the case of the SMILE technique both the lamellar incision and the lateral incision are smaller in comparison with the FS-LASIK technique, and it is therefore possible to expect damage to a lesser number of nerve endings [1].

Osmolarity of the lachrymal film is described as a significant objective indicator of dry eye syndrome. As a rule, in patients with dry eye there is an increase of osmolarity of the lachrymal film above the norm. Values of $302 \pm$ $8 \mathrm{mOsm} / \mathrm{l}$ (13) are considered to be physiological, and the value of $308 \mathrm{mOsm} / \mathrm{l}[9,13]$ is generally considered to be 
borderline. The limit value of $312 \mathrm{mOsm} / \mathrm{l}$ has a specificity of $92 \%$, upon $73 \%$ sensitivity [9]. Osmometers produced by various manufacturers are used for examining osmolarity of the lachrymal film. In comparison with certain other instruments of a similar type, the instrument TearLab ${ }^{\text {тм }}$ Osmolarity System (TearLab Corp., San Diego, CA) has high precision of osmolarity measurement [11, 14], and is often used for the detection of pathologies linked with dry eye syndrome, or in the case of other diseases linked with a change of tear osmolarity (e.g. lagophthalmos) - [12]. For examination with the aid of this instrument it is necessary to have a minimal quantity of tears -50 nanolitres, which are absorbed by a single-use testing card, in which the actual measurement is performed by the method of electric impedance.

The aim of our study was to compare the differences in preoperative and postoperative values of tear osmolarity in patients who underwent a refraction procedure of the type FS-LASIK and ReLEx SMILE.

\section{METHOD}

This concerned a prospective, non-randomised comparative trial. We evaluated the results in two groups of patients who underwent a bilateral refractive procedure for the correction of myopia and if applicable astigmatism at the Eye Centre in Horní Počernice in the period from $5 / 2014$ to $5 / 2015$. The trial incorporated two consecutive groups of patients, each of which was composed of 15 patients (30 eyes). Division of the patients into the individual groups was determined by the patients' preference, the ReLEx SMILE method is presented as a newer method, which is however financially more expensive (the difference for the patient is approx. CZK 5400 per eye). Patients in one group underwent a refractive procedure of the type of FS-LASIK. A lamella was created with the aid of a VisuMax femtosecond laser (Carl Zeiss Meditec AG, Jena, Germany), the refractive procedure itself was performed with the use of a Mel 80 excimer laser (Carl Zeiss Meditec AG, Jena, Germany). Patients in the second group underwent an operation of the type of ReLEx SMILE, performed using the instrument VisuMax (Carl Zeiss Meditec AG, Jena, Germany). In the case of all patients, the operation and postoperative course were standard, without complications. Postoperatively for 10 days the patients applied the eye drops Oftaquix (Santen OY, Tampere, Finland) and Dexamethazone (Polfa SA, Warsaw, Poland).

Bilateral measurement of osmolarity of the lachrymal film was measured in all patients with the aid of a TearLab instrument on the day of operation, as well as on the first day after surgery, one month and 3 months after surgery. The instrument was calibrated according to the manufacturer's instructions at the beginning of each examining day, and both eyes were measured together, the right eye first, without the use of topical or other anaesthesia.

The exclusion criteria for patients in the trial were serious pathology of the ocular surface in anamnesis, acute inflammation of the eye, active ocular allergy, previous other surgical procedure on the eye, use of other eye drops beyond the standard postoperative protocol, use of contact lenses less than 1 month before the operation, patients after occlusion of lachrymal puncta, with evident deformity of the eyelid or with pathological function of the eyelids, with abnormal function of the lachrymal pathways. Of general pathologies, patients with a disorder of the thyroid gland, diabetes and other general pathologies which may influence the production of tears, as well as pregnant and breastfeeding patients, patients using general medications which may influence the function of the lachrymal glands, including hormonal contraception, were excluded from the trial.

The patients signed an informed consent form for the operation and inclusion in the trial, the work was performed in accordance with the Helsinki Declaration of 1975, revised in the year 2000 .

The results were processed with the aid of the statistical program Microsoft Office Excel 2016 (Microsoft corp.) for PC. For comparison of the change of osmolarity preoperatively and in the postoperative periods, a Student paired ttest was used, for comparison of osmolarity between the two groups we used a Student unpaired t-test. We considered values of $p \leq 0.05$ to be statistically significant.

\section{RESULTS}

The average age of the patients in both groups was 28 years. In the group of ReLEx SMILE $28.0 \pm 6.2$ years (min. 19, max. 40), in the group of FS-LASIK $27.9 \pm 6.2$ years (min. 19, max. 41). The average preoperative value of osmolarity of the lachrymal film was practically the same in both groups, in the group of ReLEx SMILE 294.9 $\pm 13.4 \mathrm{mOsm} / \mathrm{l}$ and in the group of FS-LASIK $296.4 \pm 13.1$. The difference between the two groups was not statistically significant. In both groups of patients there was a pronounced increase in the average value of osmolarity, and subsequently a gradual reduction thereof one month and 3 months after the operation. The average values of osmolarity in both groups are presented synoptically in table 1 and graph 1 . The difference between the preoperative and postoperative value in the group of patients who underwent ReLEx SMILE was statistically significant 1 day and 1 month after surgery. Three months after surgery normalisation of values was practically achieved, and the difference was no longer statistically significant. In the group of FS-LASIK the differences were statistically significant at all the observed times, i.e. on the 1st postoperative day, one month after surgery and 3 months after surgery, even if in this group also there is an evident gradual reduction of the values over time.

If we statistically mutually compare the average values of tear osmolarity in both groups in the individual observation periods, we determine higher values of osmolarity in all the postoperative periods in patients who underwent an FS-LASIK type operation in comparison with patients following a ReLEx SMILE procedure. On the first day and month after the operation this difference was statistically significant, 3 months after surgery the difference was no longer statistically significant. 
Table 1: Average values of tear film osmolarity $(\mathrm{mOsm} / \mathrm{l})$ in patients udergoing refractive surgery.

\begin{tabular}{|l|l|l|l|l|}
\hline group & before surgery & 1 day & 1 month & 3 months \\
\hline ReLEX SMILE & $294,9 \pm 13,4$ & $311,7 \pm 13,4$ & $301,4 \pm 11,7$ & $296,4 \pm 13,3$ \\
\hline FS-LASIK & $296,4 \pm 13,1$ & $335,9 \pm 18,6$ & $320,1 \pm 14,7$ & $306,5 \pm 13,1$ \\
\hline
\end{tabular}

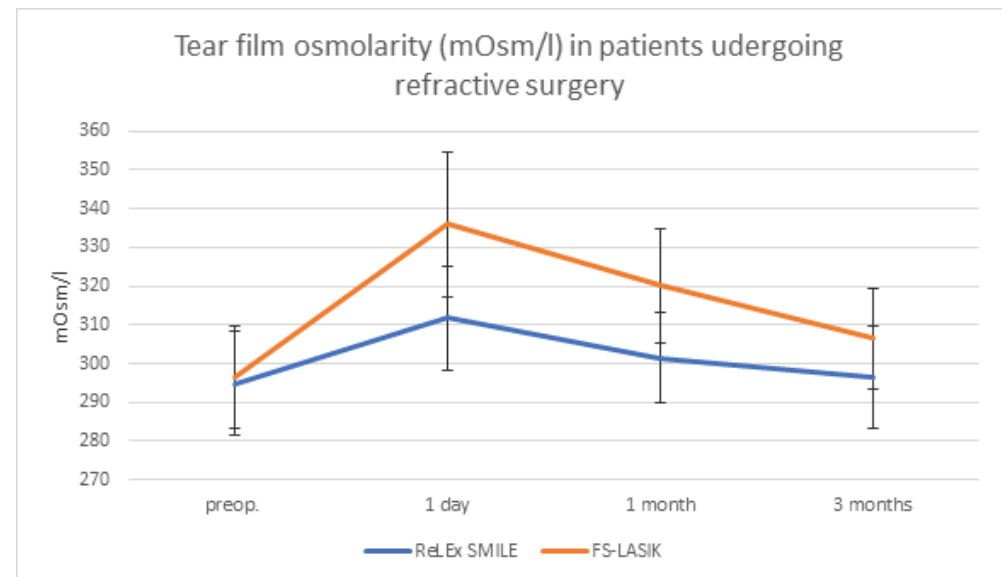

Graf. č. 1: Osmolarita slzného filmu pacientů po refrakčním zákroku

\section{DISCUSSION}

Procedures of the type LASIK and ReLEx SMILE are currently used as standard in the surgical correction of refractive errors, above all myopia. Each of these methods has its theoretical advantages and disadvantages. Upon use of the ReLEx SMILE method, a classic flap is not created, only a corneal pocket is formed, which should lead to greater stability of the cornea, lower incidence of postoperative ectasias and elimination of problems with random postoperative shift of the flap. Nevertheless, in the ReLEx SMILE method also, cases of postoperative ectasia have been described. On the other hand, the LASIK method is capable of safely correcting small dioptric defects in which the removal of a very small lenticule (in the case of the ReLEx SMILE technique) wouId be very difficult to perform. In addition, any additional correction is very difficult to resolve in the case of the ReLEx SMILE method. Another advantage of the ReLEx SMILE method is the use of a femtosecond laser, which theoreticaIly increases the speed and effectiveness, and also reduces the cost of the entire procedure. Published studies evaluating effectiveness, postoperative visual acuity and complications evaluate both methods as comparable [3], or in certain parameters (corneal sensitivity, corneal hysteresis, higher order aberrations) prefer the ReLEx SMILE method [7, 22].

Nevertheless, in a range of published studies, problems with dry eye are described as one of the most frequent postoperative complications in the case of both of the aforementioned methods. A number of studies have compared manifestations of dry eye in patients following FS-LASIK and ReLEx SMILE. The evaluated factors in these studies are various parameters - Schirmer's test (ST), break-up time (BUT), colouring of cornea, corneal sensitivity, questionnaires determining subjective complaints, OSDI (ocular surface disease index). Xia determined a reduction of the value of the break-up time test (BUT) and Schirmer's test (ST) in both groups, but the reduction was more pronounced in the FS-LASIK group [22]. Also Ganesh in his study states a higher incidence of complaints with dry eye in patients following LASIK [7]. Wang demonstrated that ReLEx SMILE has a lesser influence on the development of symptoms of dry eye, and a quicker return to normal values of the lachrymal film. The results of the subjective questionnaire and BUT were not statistically significant one month after the procedure, but in the subsequent periods (3, 6 and 12 months after surgery) the BUT values were higher in the ReLEx SMILE group [21]. Xu determined significantly lower values of ST 3 and 6 months after surgery in the LASIK group. In this study BUT was also significantly lower in the LASIK group, 1 month after surgery [23]. Li in his study determined a reduction of BUT in both groups. Nevertheless, in patients after a ReLEx SMILE procedure, spotted colouring of the cornea with fluorescein occurred less frequently, and corneal sensitivity in the central region was also higher in this group of patients [10].

Shen conducted a meta-analysis from the published studies, into which he incorporated the results of 6 published studies ( 5 cohort and 1 randomised control study) comparing the influence of the ReLEx SMILE and LASIK methods on complaints and symptoms in connection with dry eye. In total this meta-analysis compared the results of 291 eyes after ReLEx SMILE and 277 eyes after FS-LASIK. The authors did not determine a statistically significant difference in the quality of the lachrymal film upon examination using ST, nevertheless the BUT values in the first, third and sixth month after surgery were significantly worse in the FS-LASIK group. In addition the OSDI (ocular surface disease index) at these time intervals after surgery was statistically significantly worse in the FS-LASIK group. Six months after surgery the BUT and osmolarity values returned to the preoperative values in both groups of patients, the OSDI value was also nor- 
malised in the group of patients who had undergone ReLEx SMILE. The ST value remained worsened in both groups, in the FS-LASIK group the OSDI also remained worsened [17].

Only two studies available to us (source - www.pubmed. com, as of 1.12.2017) have compared the osmolarity of lachrymal film in patients after FS-LASIK and ReLEx SMILE. Denoyer et al. determined average osmolarity in a group of patients treated with FS-LASIK one month after surgery with a value of $316.3 \pm 11.6 \mathrm{mOsm} / \mathrm{l}$ and 3 months after surgery $315 \pm 11.9 \mathrm{mOsm} / \mathrm{l}$, in a group treated with ReLEx SMILE $305.1 \pm 12.5$ and $300.3 \pm 11.4 \mathrm{mOsm} / \mathrm{I}$ respectively. The differences between the groups were statistically significant in both observed periods (6). By contrast Demirok did not determine a significant difference in postoperative osmolarity between the two groups - FS-LASIK $302 \pm 10$ and $304 \pm 8 \mathrm{mOsm} / \mathrm{l}$, ReLEx SMILE $303 \pm 10$ and $306 \pm 9$ $\mathrm{mOsm} / \mathrm{l}[5]$.

In our study also we concentrated on the objective change of osmolarity of the lachrymal film as a possible indicator of postoperative complaints with dry eye. In our group there was an increase in both groups of patients, nevertheless the increase of osmolarity was statistically significantly higher in the FS-LASIK group. Over the course of 3 months there was a progressive return to the original values in both groups, in the FS-LASIK group the osmolarity value was statistically significantly higher also 3 months after surgery. In our opinion this finding supports the hypothesis of lesser damage to nerve endings during the course of a ReLEx SMILE operation in comparison with the FS-LASIK method.

A relative limitation of our study was the relatively small sample of patients and the non-randomised distribution of patients into individual groups, which was due to the different price of the procedure at our centre.

\section{CONCLUSION}

In our cohort of patients following the performance of a refractive procedure by the FS-LASIK method we registered a higher average value of osmolarity of the lachrymal film in comparison with patients after a procedure of the ReLEx SMILE type, in all the observed periods ( 1 day, 1 month and 3 months after surgery). We consider a procedure of the ReLEx SMILE type to be a method with a lesser influence on the quality of the lachrymal film, with a quicker return to the original values of osmolarity and potentially greater comfort for the patient
1. Ağca, A., Demirok, A., Yıldırım, Y., et al.: Refractive lenticule extraction (ReLEx) through a small incision (SMILE) for correction of myopia and myopic astigmatism: current perspectives. Clin Ophthalmol, 10; 2016: 1905-1912.

2. Ambrósio, R. Jr., Tervo, T., Wilson, SE.: LASIK-associated dry eye and neurotrophic epitheliopathy: pathophysiology and strategies for prevention and treatment. J Refract Surg, 24; 2008: 396-407.

3. Ang, M., Tan, D., Mehta, JS.: Small incision lenticule extraction (SMILE) versus laser in-situ keratomileusis (LASIK): study protocol for a randomized, non-inferiority trial. Trials, 31; 2012: 13:75.

4. Battat, L., Macri, A., Dursun, D., et al.: Effects of laser in situ keratomileusis on tear production, clearance, and the ocular surface. Ophthalmology, 108; 2001: 1230-5.

5. Demirok, A., Ozgurhan, EB., Agca, A., et al.: Corneal sensation after corneal refractive surgery with small incision lenticule extraction. Optom Vis Sci, 90; 2013: 1040-7.

6. Denoyer, A., Landman, E., Trinh, L., et al.: Dry eye disease after refractive surgery: comparative outcomes of small incision lenticule extraction versus LASIK. Ophthalmology, 122; 2015: 669-76.

7. Ganesh, S., Gupta, R.: Comparison of visual and refractive outcomes following femtosecond laser- assisted lasik with smile in patients with myopia or myopic astigmatism. J Refract Surg, 30; 2014: 590-6.

8. Lee, JB., Ryu, CH., Kim, J., et al.: Comparison of tear secretion and tear film instability after photorefractive keratectomy and laser in situ keratomileusis. J Cataract Refract Surg, 26; 2000: 1326-31.

9. Lemp, MA., Bron, AJ., Baudouin, C., et al.: Tear osmolarity in the diagnosis and management of dry eye disease. Am J Ophthalmol, 151; 2011: 792-798.

10. Li, M., Zhao, J., Shen, Y., et al.: Comparison of dry eye and corneal sensitivity between small incision lenticule extraction and femtosecond LASIK for myopia. PLoS One, 8; 2013; e77797

11. Nolfi, J., Caffery, B.: Randomized comparison of in vivo performance of two point- of-care tear film osmometers. Clin Ophthalmol, 11; 2017: 945-950.

12. Píšová, A., Chovanec, M., Betka,
J., et al.: Osmolarita slz u pacientů s lagoftalmem. Čes. a slov. Oftal, 72; 2016: 172-176.

13. Potvin, R., Makari, S., Rapuano, CJ.: Tear film osmolarity and dry eye disease: a review of the literature. Clin Ophthalmol, 9; 2015: 2039-2047.

14. Rocha, G., Gulliver, E., Borovik, A., et al.: Randomized, masked, in vitro comparison of three commercially available tear film osmometers. Clin Ophthalmol, 11; 2017: 243-248.

15. Savini, G., Barboni, P., Zanini, M., et al.: Ocular surface changes in laser in situ keratomileusis-induced neurotrophic epitheliopathy. J Refract Surg, 20; 2004: 803-9.

16. Sekundo, W., Kunert, KS., Blum, M.: Small incision corneal refractive surgery using the small incision lenticule extraction (SMILE) procedure for the correction of myopia and myopic astigmatism: results of a 6 month prospective study. $\mathrm{Br} \mathrm{J}$ Ophthalmol, 95; 2011: 335-9.

17. Shen, Z., Zhu, Y., Song, X., et al.: Dry Eye after Small Incision Lenticule Extraction (SMILE) versus Femtosecond Laser-Assisted in Situ Keratomileusis (FS-LASIK) for Myopia: A Meta-Analysis. PLoS One.11; 2016: e0168081 
18. Shin, SY., Lee, YJ.: Conjunctival changes induced by LASIK suction ring in a rabbit model. Ophthalmic Res, 38; 2006: 343-9.

19. Shtein, RM.: Post-LASIK dry eye. Expert Rev Ophthalmol, 6; 2011: 575-582.

20. Solomon, R., Donnenfeld, ED., Perry, HD.: The effects of LASIK on the ocular surface. Ocul Surf, 2; 2004: 34-44.
21. Wang, B., Naidu, RK., Chu, R., et al.: Dry Eye Disease following Refractive Surgery: A 12-Month Follow-Up of SMILE versus FS-LASIK in High Myopia. J Ophthalmol, 2015;2015:132417

22. Xia, L., Zhang, J., Wu, J., et al.: Comparison of Corneal Biological Healing After Femtosecond LASIK and Small Incision Lenticule Extraction
Procedure. Curr Eye Res, 41; 2016: 1202-8.

23. Xu, Y., Yang, Y.: Dry eye after small incision lenticule extraction and LASIK for myopia. J Refract Surg, 30; 2014: 186-90.

24. Yu, EY., Leung, A., Rao, S.,et al.: Effect of laser in situ keratomileusis on tear stability. Ophthalmology, 107; 2000: 2131-5. 